Cahiers de recherches médiévales

\title{
La sainteté à l'épreuve de la croisade
}

La Vie de saint Louis de Jehan de Joinville

\section{Marie-Geneviève Grossel}

\section{(2) OpenEdition}

Journals

Édition électronique

URL : https://journals.openedition.org/crm/2521

DOI : $10.4000 / \mathrm{crm} .2521$

ISSN : 1955-2424

Éditeur

Honoré Champion

Édition imprimée

Date de publication : 15 janvier 1996

Pagination : 129-146

ISSN : 1272-9752

Référence électronique

Marie-Geneviève Grossel, "La sainteté à l'épreuve de la croisade », Cahiers de recherches médiévales

[En ligne], 1 | 1996, mis en ligne le 07 février 2008, consulté le 15 décembre 2022. URL : http:// journals.openedition.org/crm/2521; DOI : https://doi.org/10.4000/crm.2521 


\section{La sainteté à l'épreuve de la croisade : La Vie de saint Louis de Jehan de Joinville}

Après avoir consacré de brûlantes et enthousiastes pages à l'esprit de croisade qui marqua les premiers départs, Alphandéry et Dupront ${ }^{1}$, lorsqu'en leur beau livre ils en viennent à parler des dernières croisades, énoncent ce constat désabusé et définitif : il n'y a plus en ces années-là qu'une

série d'opérations, de mariages, d'espoirs de succession qui montrent combien à ce moment le sens mystique de la croisade, dans ses fins providentielles, du royaume de Jérusalem, de l'héritage du Christ, est obnubilé ou complètement perdu.

Néanmoins, le plus souvent, on n'accuse pas saint Louis d'avoir manqué de sens mystique mais bien plutôt d'avoir conçu d'une façon déjà anachronique sa mission de croisé, terminée par l'échec irrémédiable que l'on sait. Qu'en pensèrent donc ceux qui l'accompagnaient? Pour l'un d'entre eux au moins, Jean de Joinville $^{2}$, nous croyons percevoir l'écho perdu d'une conviction ou d'une croyance; mais il faut naturellement ne jamais oublier que celui qui écrit - qui récrit une histoire vécue - est déjà bien loin de celui qui se trouvait aux côtés d'un roi dont chaque page de son livre proclame combien il l'aimait ${ }^{3}$. Nous tenons ainsi un témoignage, ou un jugement, porté sur la croisade par un homme du début du XIVe s., chargé d'une lourde expérience. Et derrière les pensées de Joinville, on trouve celles qu'il attribue au roi, sans doute elles aussi en partie reconstruites, à travers le miroir doublement déformant de l'altérité et du temps. Enfin, outre la conception de Joinville ou celle qu'il prête à son royal ami, ne pouvons-nous discerner un certain idéal de roi, saint, croisé, martyr, tel que la canonisation l'a figé pour la postéritée nouveau David dont la sainteté se construirait à l'épreuve du pélerinage sanctionné par la mort?

\section{I - Joinville devant sa croisade.}

Si Joinville nous décrit avec beaucoup de détails, et aussi avec une habileté consommée, ses préparatifs et son départ pour la Terre Sainte, on remarquera qu'à

1 P. Alphandéry et A. Dupront, La chrétienté et l'idée de croisade, 2 vol. , Paris, 1954-1959, citation p. 203.

2 Nous citons Jean de Joinville dans l'édition de Natalis de Wailly, Histoire de saint Louis, Paris, 1884, beaucoup plus riche que celle de N. Corbett, La vie de saint Louis, Quebec, Canada, 1977, que nous avons cependant consultée également.

3 Cf. . M. Zink, «Joinville ne pleure pas mais il rêve», Poétique, T. 33, 1978, pp. 28-45

4 Voir les intéressantes suggestions à ce propos de J. Marot-Lassauzaie, L'image de saint Louis dans la littérature du Moyen Age, thèse de troisième cycle, Nice, 1979

Cahiers de Recherches Médiévales (XIIf-XV s.), 1, 1996 
nul moment il n'éprouve le besoin de nous expliquer pourquoi il partit.Le texte raconte la maladie du roi puis sa prise de croix mais n'indique pas davantage les motifs qui poussèrent saint Louis :

Nostre Sires ouvra en li et li envoia santei tantost ; car il estoit emuyz et ne pouoit parler. Et si tost qu'il fu en estat pour parler, il requist que on li donnast la croiz et si fist on (\$107).

Mais le parallélisme ainsi tracé subtilement entre les deux héros de l'histoire ne nous empêche pas de supposer que, pour Joinville, la croisade apparaissait si bien comme une tradition familiale qu'il suivit l'élan d'un roi qu'il appréciait déjà, sans trop se poser de questions. L'on connaît l'admiration que le sénéchal portait à son oncle Geoffroi de Joinville, mort au Krak des Chevaliers et dont notre sénéchal écrivit lui-même l'épitaphe ; en outre, le père de Joinville, Simon, homme pourtant bien plus mû par ses intérêts que par des élans mystiques, s'était embarqué et trouvé sous Damiette, en $1219^{5}$.

Saint Louis convoqua tous ses barons et Joinville, dont il connaissait sans doute déjà les projets ; mais Joinville ne porta pas le serment de fidélité demandé puisqu'il n'était pas le vassal du Capétien. Et dans les paragraphes suivants, il s'attache à nous retracer ses préparatifs en compagnie de ses deux cousins, sur le départ également, Gobert d'Apremont et le comte de Sarrebrück : nous restons en famille.

Pourtant si Joinville se refuse à expliciter pour son lecteur les motifs profonds qui le poussèrent au départ, alors qu'il détaillera en 1267 les raisons de son refus, nous leur découvrons un sens que le récit du départ fait plus que nous suggérer.

Joinville ne se trouve pas avec le gros des troupes royales. Il ne les rejoindra qu'à Chypre. Aussi ne saurons-nous rien des circonstances du départ de Louis, tandis que le sénéchal quittant son païs nous est proposé comme le modèle même de l'Homo Viator. Ayant conclu ses affaires avec une courtoisie, et une délicate justice que le texte souligne implicitement, notre Champenois se met dès l'orée de l'aventure sous l'égide de la sainteté : il envoie quérir l'abbé de la proche abbaye de Cheminon, car un jour de fête de Notre Dame à Clairvaux, où précisément Louis était présent, Joinville s'était vu désigner ce cistercien comme «li plus preudom qui soit en toute l'ordre blanche» ( $\$ 120)$ Et l'interlocuteur de Joinville de lui confier que ce moine était l'objet de miracles pleins de sollicitude de la part de la Vierge :

J'ai oy conter a un preudome qui gisoit ou dortour là ou li abbes de Cheminon dormoit : et avoit li abbes descouvert sa poitrine pour la grant chalour que il avoit ; et vit cis preudom, qui gisoit ou dortour ou li abbes de Cheminon dormoit, la Mere Dieu qui ala au lit de l'abbei, et li retira sa robe sur son piz pour ce que li vens ne li feist mal (\$121).

Voilà donc sous quelle protection s'opère le départ béni de Joinville, en la présence d'un saint à laquelle répond,tout de même, au retour de saint Louis à Hyère la bénediction d'un autre religieux, le cordelier frère Hugues, dont le sermon porte sur la justice du roi, deçà la mer cette fois. Hugues est le fameux saint archevêque de

5 H. F. Delaborde, Jean de Joinville et les seigneurs de Joinville, Paris, Picard, 1894. 
Digne, frère de la béguine Douceline ${ }^{6}$. A l'heure où Joinville écrit Dieu a dûment sanctionné sa sainteté : "Or m'a l'on puis dit que il gist en la citéi de Marseille là où il fait mout beles miracles» ( $\$ 660$ ).

Le départ de Joinville est donc une évidente démonstration de la valeur de son engagement et de la justesse de sa cause.Tout son livre visant à souligner la grandeur du voeu de croisade royal, le sénéchal, ainsi qu'on l'a noté, construit en contrepoint sa propre démarche, tandis qu'absence et présence s'entrecroisent comme des fils dans la trame. Omniprésence dès l'ouverture de ce thème de la sainteté : Joinville au beau milieu des fastes du festin de Saumur n'omet pas de préciser que tel jeune Allemend de 18 ans qui sert la table de la reine Blanche «avoit estei fiz sainte Helisabeth de Thuringe, que l'on disoit que la royne Blanche le besoit ou front par devocion pour ce que elle entendoit que sa mere l'i avoit maintes foiz besié» (\$96).

Les gestes que capte l'œil attentif du jeune écuyer et qu'il gardera en sa fidèle mémoire sont pour lui lourds de signification. Ainsi encore du geste de saint Louis lavant, aux jeudis saints, les pieds des «pauvres», de façon un peu théâtrale, devant un Joinville assez circonspect ; et, sentant peut-être ces réticences, le roi de lui demander s'il en ferait autant : "Sire, dis-je en maleur! les piez de ces vilains ne laverai je jà!» (§ 29). Derrière la vivacité de l'anecdote, comment ne pas reconnaître le texte même de l'Evangile ? Nul doute que saint Louis, lavant les pieds des mendiants, ne suivît à la lettre l'exemple du Christ ; mais, bien entendu, le refus spontané de Joinville le met, pour sa part, dans le rôle de saint Pierre! Et c'est bien ainsi que fut conçue cette Vie de saint Louis ; en face d'un roi qui voulait suivre absolument le Christ, ainsi que Joinville l'énonce en toutes lettres, le sénéchal, témoin et zélé propagandiste, se veut l'apôtre. C'est dire qu'il revendique pour lui aussi un peu de la sainteté de son ami, comme si elle avait diffusé sur lui, telle Blanche la trouvant sur le jeune front du fils d'une sainte, et plus encore, si l'on prend garde au nombre de fois où Joinville rappelle à plaisir combien Dieu lui fut sans cesse présent, le protégeant et le guidant.

C'est même en ce sens que l'on pourrait interpréter le récit fameux dans lequel Joinville, ayant raillé levilain Robert de Sorbon sur sa vêture, se voit remis en place par Louis IX, puis un peu plus tard, admis en sa royale intimité, se retrouve assis, près à le toucher, de son roi qui le console. Aux pieds de Louis, son fils et son gendre, le tout se déroulant juste après un repas, un jour de Pentecôte. Derrière cette scène, ne voit-on pas se profiler une autre Cène, où le disciple aimé s'appuyait contre le sein de son Messie pour s'entendre chuchoter une vérité à lui seul réservée? Car avec Jean, Joinville partagea la vénération absolue de la jeunesse envers un Maître follement admiré et chéri, le goût de l'écriture et du témoignage, jusqu'à la longévité, propice à la remémoration douce-amère d'une longue survie à l'aimé. Et surtout le texte entier de Joinville veut nous faire voir en ce jeune et ardent sénéchal, comme pour le disciple que Jésus aimait, l'intime, le préféré.

Ainsi donc pour qui imite le Christ, pour qui se veut le Disciple, le passage vers l'Outremer paraît aller de soi : mettre ses pas dans les pas de l'Homme-Dieu, trajet

6 Cf. R. Gout, La vie de sainte Douceline, Paris, 1927. 
déjà immémorial depuis Egérie ${ }^{7}$, et avant elle. Egérie, toutefois ne portait pas l'épée... Joinville et saint Louis, si.

Certes, les papes s'étaient depuis longtemps préoccupés de définir la différence entre le simple pélerin et le croisé : le votum crucis, le subsidium Terrae Sanctae permettaient au pélerin de coeur d'être guerrier de fait. Mais le croisé recevait à son départ la bénediction reservée au pélerin ${ }^{8}$ et, notons-le, Joinville qui évoque son départ (§ 122) «nus piés, deschaus, en langes», son bourdon et son escharpe, ne nous parle pas de sa prise de croix proprement dite, alors qu'il le fait pour le roi.. Il semblerait que la responsabilité de la croisade soit ainsi laissée à son chef ; simple soldat, Joinville obéit - avec vaillance, toutefois ; mais le but de l'expedition, ses succès ou défaites, ressortissent du seul roi. Avoir participé à la septième croisade se définira donc pour Joinville comme «avoir estẻ au servise Dieu et le roy outremer». Servise ici a son plein sens féodal de «service armé». Mais c'est esquiver une difficulté que soulignent cruellement les vassaux du roi lors de la seconde prise de croix de mars 1267 : force leur est de choisir entre le «service de Dieu» ou celui du roi car les deux ne sont pas du tout naturellement complémentaires :

Se li roys se croise, ce yert une des douloureuses journées qui onques fust en France. Car se nous ne nous croisons, nous perderons le roy ; et se nous nous croisons, nous perderons Dieu, que nous ne croiserons pas pour li mais pour paour du roy. (\$ 733)

Nous sommes ici bien loin de Rutebeuf qui, lorsqu'il exhorte à aller «Vengier La honte Dieu outremer» ${ }^{9}$, (terme que Joinville n'emploie jamais), présente pour son décroisé des arguments d'assez bas étage : aventure ruineuse et vaine, la croisade doit être renvoyée aux clercs, les premiers concernés, et qu'on laisse chaque seigneur vivre en paix chez lui à son aise!

Clerc et prelat doivent vengier

La honte Dieu, qu'il ont sa rente ${ }^{10}$.

Pour le simple seigneur que se veut Joinville, la croisade se déroule entre deux idéaux : elle est profondément aimantée par le modèle épique et la traverse de part en part l'aspiration à la mort glorieuse du «martyre».

Que la chanson de geste soit l'un des intertextes de la Vie de saint Louis, c'est une évidence qu'avait jadis soulignée K.Uitti ${ }^{11}$ et qu'évoque aussi en passant $D$. Boutet dans son livre sur le «roi imaginaire» ${ }^{12}$ : la même sainte cause mène un Charlemagne et le chef des croisés; et à annoncer dans le prologue que le livre va

7 Cf. Egérie, Journal de voyage (Itinéraire) et lettre sur la Bse Egérie, éd. P. Maraval et M. C. Diaz y Diaz, Paris, Cerf, 1982.

8 Cf. article «CROISADES», Encyclopedia Universalis.

9 «La complainte de Monseigneur de Sergines», Euvres complètes de Rutebeuf, publiées par

E. Faral et J. Bastin, Paris, Picard, 1977, tome 1, p. 416, v. 9495.

10 «La desputoison du croisé et du décroisé», ibidem, p. 475, v. 129-130.

11 K. D. Uitti, «Nouvelle et structure hagiographique», Mittelalterbilder als neuer Perspektive, Munich, 1985, pp. 380-395.

12 D. Boutet, Charlemagne et Arthur ou le roi imaginaire, Paris, Champion, 1992. 
exalter les «hauts faits» et la chevalerie du saint roi, Joinville ne fait qu'accentuer le motif. L'auteur est toujours ravi de nous retracer les exploits de ses compatriotes, qu'il s'agisse de Gautier d'Autrèche, de Geoffroi de Sergines ou de Gautier de Châtillon ou encore de son prêtre personnel, aussi vaillant que dévot. Un seul exemple illustrera suffisamment ce point.

Lorsque les troupes du soudan de Damas passent devant Acre, un «chevalier de Gennes qui avoit a non monseignor Jehan le Grand» va s'illustrer par «trois beaux coups» devant la ville ; le premier lui est proposé par un Sarrasin :

Li commença a escrier en sarrazinois que il jousteroit a li se il vouloit. Et cil li dist que si feroit il volentiers $(\$ 548$.

Le duel se révelera en fait un combat contre un «tropel de Turs» dont le valeureux Jehan défait glorieusement trois cavaliers, l'un après l'autre.

Et ces trois biaus cos fist il devant le signour d'Arsur et les riches homes qui estoient en Acre et devant toutes les femmes qui estoient sus les murs pour veoir celle gent $(\S 550)$.

Notons en passant que Jehan le Grand comprenait visiblement le sarrazinois, les difficultés de compréhension dues à la langue ont peut-être parfois arrêté Joinville mais très souvent il signale la présence des drugements. Pour en revenir à la prouesse du chevalier de Gennes, on aura ici reconnu le motif cher à la geste qu'on pourrait appeler «les dames aux créneaux». Cependant vérité et fiction se nourrissent réciproquement si l'on en croit les regards acerbes que porta, depuis les murailles de sa ville, la critique Anne Comnène sur les croisés assemblés au pied des remparts; lesquels, de leur côté, paradaient glorieusement devant les belles Grecques dans la naïve conviction d'être fort admirés...

Plus profondément, ce qui donne au récit de Joinville une tonalité épique, c'est le rappel régulier de la présence de Dieu aux côtés des croisés. Comme pourraient-il n'être pas dans leur droit puisque la main du Père les guide continûment? Ainsi, lors de la dure bataille au bord du Nil, Charles d'Anjou, chargé de la défense des chats-châteaux, manque enrager lorsque le feu grégeois les détruit; pourtant ce dommage immédiat révèle un dessein de protection divine car si ces événements s'étaient produits la nuit suivante, les gardiens auraient brûlé avec les machines de guerre :

Dont il avint ainsi que nostre dui chastel furent ars ; dont li roys de Sezile estoit si hors du sens que il se voloit aler ferir ou feu pour estaindre. Et se il en fu courouciez, je et mi chevaliers en loames Dieu; car se nous eussiens guieté le soir, nous eussiens estei tuit ars. (\$210)

Entre le Dieu dont on foule la terre et ses hommes d'armes circule un courant d'affection réciproque, Dieu accorde à ses hommes sa grâce qui est salut, au sens premier du terme :

Or disons donc que grant grace nous fist Diex li touz puissans quant il nous deffendi de mort et de peril a l'ariver... Grant grace nous fist Nostre Sires de Damiette que il nous delivra, laquel nous ne deussiens pas avoir prise sanz affamer. ( $\$ 165)$ 
Le roi Jean de Brienne, sous lequel avait combattu Simon de Joinville, n'avaitil pas dû pour sa part réduire Damiette par la famine? Grâce, certes, mais aussi bien pour la croisade de 1219 que pour celle ici racontée, ces grâces divines valent-elles vraiment d'être rappelées quand on sait combien ces victoires furent sans lendemain? Ce qui reste certain, c'est que Dieu utilise pour se faire comprendre des croisés un langage qui leur est directement perceptible : «Ceste grant courtoisie fist Diex a moy et a mes chevaliers, car nous eussiens le soir guietié en grant peril.» (\$213). Ailleurs la courtoisie céleste devient un honneur: "Grant grace, fist il, devons a Nostre Signour de ce qu'il nous a fait tiex dous honnours en ceste semainne.» ( $\$$ 279). Et l'honneur est bien ce qui meut le sénéchal lorsqu'on l'invite à rétorquer à ceux qui croient l'insulter en le traitant de poulain: «que je me defendisse vers ceus qui $\mathrm{m}$ 'apeloient poulain et leur deisse que $\mathrm{j}$ 'amoie miex estre poulains que roncins recreus aussi comme il estoient.» (\$434).

Temps de l'épreuve donc, temps où l'on se découvre digne ou indigne de l'honneur de Dieu, savoir adorer les dons du ciel savoir interpréter ses «menaces» comme le rappelle le roi à son ami $(\S 39 \mathrm{sq})$. Mais l'épreuve la plus gratifiante et la plus méritoire est bien celle qui pousse le témoin jusqu'à l'offrande suprême, celle de son existence. Depuis longtemps, la mort pour la Terre Sainte avait été assimilée au martyre ; les chants de croisade ne cessaient de répéter qu'» on doit Paradis Conquerre par mesaise avoir» ${ }^{13}$; on voyait aussi dans la mort sur le champ de bataille une application quelque peu déviée du précepte christique: «ll n'y a pas de plus grand amour que de donner sa vie pour celui que l'on aime». Dieu était ici le destinaire du sacrifice en même temps que l'Ami.

Joinville montre bien que cet idéal entraînait certains de ses amis, ainsi du celerier de Doulevens, qui, sur le moment de se rendre, exprime une tout autre conviction :

«Sire, je ne m'acort pas a cest consoil» Je li demandai auquel il s'acordoit et il me dist «Je m'acort que nous nous lessons touz tuer, si nous en irons tuit en Paradis». ( $\$$ 319)

Mais justement, ajoute Joinville, «nous ne le crûmes pas». Le sénéchal avait d'ailleurs pris soin de nous laisser entendre que, puisque le roi était déjà pris, on pouvait estimer providentiel le vent contraire qui les arrêta sur le fleuve, les contraignant eux-aussi à la reddition, c'est-à-dire aux retrouvailles avec le roi.

Pourtant, est-ce nostalgique admiration ou question restée sans réponse à sa propre survie? Joinville s'étend sur les martyres volontaires de ceux qui coururent, le coeur joyeux, à l'épreuve suprême, Dieu les exauçait en leur accordant la palme. Ainsi du sire de Brancion: grièvement blessé au bord de la rivière avec ses vingt chevaliers dont douze allaient succomber là, il ne survécut pas à cette journée. Mais jadis ce seigneur de Bourgogne avait participé à une bagarre contre des Allemands, coupables d'avoir occupé une abbaye ; et, les ayant chassés et tués, il s'était écrié avec douleur:

13 Voir Les chansons de croisade éditées par J. Bédier et P. Aubry, Genève, Reprints, 1974, la citation vient de la ch. 55, v. 23-24 de Thibaut de Champagne (Les chansons de Thibaut de Champagne, roi de Navarre, éd. par A. Wallensköld, Paris, SATF, 1925). 
«Sire, je te pri que il te preingne pitié de moy et m'ostes de ces guerres entre crestiens là où j'ai vecu grant piesce; et m'otroies que je puisse mourir en ton servise par quoi je puisse avoir ton regne de paradis» Et ces choses vous ai je ramenteu por ce que je croi que Diex li otroia. (\$ 278).

La mort frappe tout autour de Joinville, le martyre est réservé à ses proches, tel Gautier de Brienne, suzerain et parent de Joinville: sa mort dans les supplices est tout à fait digne des saints des premiers temps :

Avant que li emperieres de Perse alast devant La Chamelle, il amena le conte Gautier devant Jaffe ; et le pendirent par les bras a unes fourches et li dirent que il ne le despenderoient point jusques a tant que il averoient le chastel de Jaffe. Tandis que il pendoit par les bras, il escria a ceus dou chastel que pour mal que il li feissent, que il ne rendissent la ville, et que se il la rendoient, il meismes les occiroit. (\$ 536)

Utilisé comme monnaie d'échange entre les soudans, Gautier finira assassiné en sa prison, longtemps avant que Joinville ne récupère et n'ensevelisse pieusement ses restes: «Et il l'alerent occire en la prison et martyrier; dont nous devons croire qu'il est es ciex ou nombre des martirs.» (\$ 538).

Mais Joinville que sa foi ne poussait pas aux folies exaltées des saints, qui ne désirait pas être de coeur pur mais de corps lépreux, remercie plutôt Dieu de l'avoir préservé de tous les périls. Le martyre est glorieux lorsqu'il frappe ceux qui l'ont désiré :

Jaque de Castel, evesque de Soissons. Quant il vit que nos gens s'en revenoient vers Damiette, il qui avoit grant desirier de aler a Dieu ne s'en vout pas revenir en la terre dont il estoit nez; ainçois se hasta d'aler avec Dieu et feri des esperons et assembla aus Turs touz seux qui a lour espées l'occistrent et le mistrent en la compaingnie Dieu ou nombre des martirs. (\$ 393)

Joinville admire mais il n'imite pas. Peut-être même reste-t-il secrètement critique lorsqu'il rapporte les brutales paroles de l'Hospitalier Henri de Ronnai à saint Louis s'enquérant de son frère porté disparu : Et il li di qu'il en savoit bien nouvelles car estoit certeins que ses freres li cuens d'Artois estoit en Paradis. ( $\$ 244)$. Et ce moine-soldat quelque peu soudard enchaîne aussitôt sur le «si grans honnours» que Dieu a fait au roi en lui permettant de se tirer au moindre mal d'une défaite accablante.Quant aux morts, n'en parlons plus et Dieu ait leur âme! Et saint Louis d'acquiescer à cette rude et déchirante philosophie. Mais Joinville, attentif plutôt aux larmes échappées à son roi, avait clairement indiqué auparavant que le passage par l'Egypte était le choix de Robert d'Artois, accepté par le roi contre l'avis du Conseil, que c'est le même Robert qui porte l'entière responsabilité du carnage de la Mansourah.

On peut donc conclure que, tout comme Joinville ne nous donnait pas les raisons de son départ outremer, laissant à son roi la responsabilité du passage qu'il avait suivi par admiration personnelle et tradition familiale, l'idée que la croisade est une épreuve sanctifiante que couronne un volontaire martyre nous est présentée avec certaines ambiguités. Admirable folie réservée aux saints plutôt que modèle ? Nous n'aurons pas de réponse mais la question est significative. 
II - Le roi et la croisade.

La dignité royale que Joinville incarne en son ami se donne pour but essentiel le bien du royaume, le profit d'un peuple. C'est, dès le prologue, la plus éminente qualité que le sénéchal concède à son roi :

Li secons livres vous parlera de ses granz chevaleries et de ses granz hardemens liquel sont tel que je li vi quatre foiz metre son cors en avanture de mort aussi comme vous orrés ci après pour espargnier le doumaige de son peuple. (\$6).

Or tout semble se passer comme si, dans le récit de Joinville, saint Louis estimait diriger deux royaumes, celui de France et celui de Jérusalem dans lequel, une fois libéré de sa captivité, il s'installe en maître absolu, n'hésitant pas, par exemple, à punir d'un exil sans retour tel Templier qui lui avait désobéi et dont la présence aurait sans doute été utile à la Terre Sainte, une fois terminé le séjour temporaire du roi ( $\$ 512 \mathrm{sq}$ ).Voilà donc sous la plume du sénéchal une justification à la croisade de saint Louis, qu'il peut déduire d'une existence à présent achevée : «Son cors mist il en avanture pour le peuple de la terre garantir qui eut estéi perdus dès lors se il ne fust lors remez.» (\$12). Assurément, après l'échec de sa croisade armée, tout semblait perdu si le roi quittait l'Outremer; mais il n'est pas évident que l'état du Royaume de Jérusalem était vraiment pire avant que la croisade n'ait eu lieu... Quoi qu'il en soit, lors des fameuses délibérations à Acre concernant le séjour ou le départ, saint Louis pose de façon fort claire l'alternative qui s'offre à lui :

Signour, ma dame la royne ma mère m'a mandéi et prié... que je m'en voise en France car mes royaumes est en grant peril... Cil de ceste terre a cui j'en ai parlei m'ont dit que se je m'en voi, ceste terre est perdue. Car il s'en venront tuit en Acre après moy, pour ce que nulz n'i osera demourer a si pou de gent. (\$ 419).

La réponse de son conseil est non moins claire : qu'il s'en aille en son royaume car il y va de son honneur, mais qu'il y rassemble des troupes et de l'argent pour une future et proche croisade qui serait une juste vengeance :

Vous n'avez pooir de demourer en cest païs a l'onour de vous ne de vostre règne... Si vous loent-il, sire, que vos alez en France et pourchaciés gens et deniers par quoy vous puissés hastivement revenir en cest païs vous vengier des ennemis Dieu qui vous ont tenu en lour prison. (\$ 423).

Solution d'autant plus simple qu'elle était banale, tous les chefs précédents l'ayant, dans les faits, acceptée, bien-sûr au détriment des Etats Latins d'Outremer ; mais les résultats obtenus par le séjour de saint Louis furent-ils autre chose qu'un bref sursis? Joinville, qui écrit après la chute d'Acre en 1291, pouvait-il penser autrement? Mais le roi avait déjà pris une décision irrévocable : «Si ai regardei que a nul fuer je ne lairoie le royaume de Jérusalem perdre, lequel je sui venuz pour garder et pour conquerre.» (\$ 437). Dans une certaine mesure, on peut dire que ce séjour permit de «garder» mais assurément pas de «conquerre»; quant à Jérusalem même, que le roi se refusa d'aller visiter pour des raisons politiques (cf. ( $\$ 554$ ), on ne saurait en l'occurrence la confondre avec son «royaume». Nous avons donc une 
croisade qui est conquête de terre, consolidation d'un pouvoir, mais que ces passages distinguent radicalement du «pélerinage», adoration des lieux saints. On notera que si saint Louis refusa d'aller en pélerin dans une ville qu'il ne pouvait faire sienne, Joinville insiste sur ses propres périples en terre Sainte:

Je priai au roy que il me lessast aler en pelerinaige à Notre Dame de Tortouze la où il avoit molt grant pelerinaige pour ce que c'est li premiers autels qui onques fust fais en l'onour de la Mère Dieu sur terre. Et y fesoit Nostre Dame mout grans miracles. (§597).

Car si Joinville n'évoque nulle part la dévotion du roi à l'égard de la Vierge, il nous donne souvent des témoignages de sa propre ferveur. Il est celui qui conseille le rite propitiatoire des processions à Damiette lorsque le retard d'Alphonse de Poitiers devient vraiment inquiétant. Le samedi, la première procession se dirige vers le «Moustier Nostre Dame» dont Joinville précise: «liquex moustiers estoit fais en la mahommerie des Sarrazins et l'avoit li legas dedié en l'onnour de la Mère Dieu.» ( $\$ 181$ ). Nous n'avons pas d'autre commentaire sur cette mosquée devenue église.

C'est encore Joinville qui nous narre un miracle advenu en mer où Notre Dame de Vauvert sauva un de ses fidèles de la noyade; notre sénéchal, vivement frappé par l'événement, le fit représenter deux fois, sur les vitraux de la chapelle de Joinville et à l'église de Blécourt. ( $\$ 651$ ). Même au moment de sa mort saint Louis n'invoque pas Marie mais saint Jacques qui, étant le patron des pélerins, préside tout naturellement aux destinées de la croisade. Joinville n'avait pas manqué de le prier, lui aussi, aux pires heures de la bataille de la Mansourah, quand tous ceux qui l'entouraient étaient si grièvement blessés qu'ils moururent peu après : «Il me souvint de mon signour saint Jaque que je requis : «Biaus sire sains Jacques, aidiés moy et secourez a ce besoing». ( $§ 225$ ). Et aussitôt l'un des blessés va quérir des secours qui sauveront Joinville.Le sénéchal, sur le point d'être pris, jette au Nil ses bijoux et ses reliques; il ne nous précise pas de quels saints. Mais tombé à genoux devant son bourreau armé d'une hache danoise, il murmure «Ainsi mourut sainte Agnès». Saint Louis salue en ses dernières pensées saint Denis et saint Geneviève, mêlant une dernière fois au nom de Jérusalem le souvenir de sa capitale parisienne, présente en ses deux saints.Il semble bien que pour le roi, la Terre Promise soit avant tout tout terre du Christ et terre de la Passion, dévotion personnelle que corroborerait peutêtre le choix des reliques gardées en la Sainte Chapelle.

Tout comme Joinville, le roi en se croisant marchait sur les traces des siens, non pas de Louis VIII qui se satisfit d'une croisade en Albigeois, mais PhilippeAuguste et surtout Louis VII ; pourtant c'est la prouesse de Richard-Coeur-de-Lion qui semble avoir fait rêver les Croisés de 1260 et peut-être suscité en saint Louis une secrète envie. Par deux fois Joinville nous présente son roi comme le modèle de toute chevalerie, à Damiette lorsqu'il veut courir sus aux Sarrasins médusés :

Quant il vint a terre et il choisi les Sarrazins, il demanda quex gent c'estoient. Et on li dist que c'estoient Sarrazin. Et il mist le glaive desous s'esselle et l'escu devant li et eust couru sus aus Sarrazins se sui preudome qui estoient avec li li eussent souffert. (§162)

Mais c'est surtout dans la douloureuse journée de la Mansourah qu'éclate la bravoure du roi On sent derrière les simples mots de Joinville, alors coincé au milieu 
de mourants, tout le soulagement, tout l'émerveillement qu'il éprouva à voir soudain se dresser son souverain comme une image digne des plus beaux romans:

Vint li roys a toute sa bataille a grand noyse et a grand bruit de trompes et de macaires et se aresta sur un chemin levei. Mais onques si bel armei ne vi. Car il paroit desur toute sa gent des les espaules en amont, un heaume dorei en son chief, une espée d'Alemainngne en sa main. (\$228)

Roi guerrier donc, incarnation même de la prouesse et de la preudomie. Les épreuves de la captivité et de ses suites vont donner encore plus à Joinville l'occasion de se rapprocher du roi et de développer pour lui une fervente admiration.

La constance, la dignité dont Louis ne se départ pas durant les heures terribles de son emprisonnement sont tout à l'opposé de la versatilité de ses adversaires qui, après s'être chargés de l'assassinat du soudan, se trouvent acculés à un horrible dilemme : «Se nous occions le roy après ce que nous avons occis le soudanc, on dira que li Egypcien sont les plus mauvaises gens et les plus desloiaus qui soient ou monde.» (\$ 372). Et d'ailleurs, en tuant leur seigneur ils se sont déjà mis hors leur loi musulmane ; mais d'un autre côté : «Encore ferons nous pis se nous ne tuons le Roy quelque assureument que nous li aiens donnei car c'est li plus forz ennemis que la loi paiennime ait.» ( $\$ 373$ ). On comprendra qu'en face de tels arguments, Joinville ait tout particulièrement admiré le sens de la vérité propre à saint Louis qui le poussa à ne jamais mentir, même à ces Sarrasins perfides! Ennemis de Dieu, païens, les Turcs et Sarrasins sont présentés sous un jour plutôt noir, ce qui est une autre façon de justifier la croisade. A saint Louis sautant de sa barque pour aller pourfendre les Sarrasins de Damiette répond la boutade féroce du même roi, définissant une discussion avec un Juif comme un coup d'épée porté en plein ventre de l'interlocuteur. Cependant ces lignes terriblement évocatrices ne renferment ni toutes les convictions prêtées par Joinville au roi ni non plus celles du sénéchal.

La Terre Promise est et reste une terre de merveilles, le Nil et ses eaux changeantes, ses cargaisons d'épices, ses senteurs de Paradis, les deux sources du Jourdain, tout baigne dans le climat d'irréalité exotique que l'on retrouve, amplement développée, en l'Historia Orientalis ${ }^{14}$ Mais les descriptions de bataille autour du Nil sont bien plus épouvantables que toute la fantasmagorie orientale dans laquelle se complaît la geste romanesque; peut-on imaginer pire que l'évocation du feu grégeois :

La manière dou feu gregeois estoit teix que venoit bien devant aussi gros comme uns tonniaus de verjus et la queue dou feu qui partait de li estoit bien aussi grans comme uns grans glaives. Il faisoit tel noise au venir que il sembloit que ce fust la foudre dou ciel; il sembloit un dragon qui volast par l'air. Tant getoit grant clartei que l'on veoit aussi clair parmi l'ost comme se il fust jours pour la grant foison dou feu qui getoit la grant clartei. (§ 206).

Face à une telle horreur, même les tempêtes imprévisibles et mortelles de la mer paraissent supportables. Pour les unes comme pour l'autre, une seule parade, la

14 Jacques de Vitry, Historia Orientalis, édition de J. Bongars, Hanovre, 1611, pp. 10471115 des Gesta Dei per Francos sive Orientalium expeditionem et regni Francorum Hierosolymitana Historia. 
prière. Gautier d'Escurés la conseille à ceux qui sont sous le feu et tombent à genoux en le voyant arriver, tandis que le roi, allongé en croix sur le pont du bateau durant les tempêtes, dresse vers le ciel, pendant les jets de feu, ses mains suppliantes : «Biaus sire Diex, gardez moi ma gent!» (§ 207).

L'ennemi qui harcèle les troupes, pénétrant dans les camps la nuit pour égorger les dormeurs, jetant pierres et flammes, n'a pas vraiment de visage ; haïssable, il l'est ni plus ni moins que tous les ennemis de toute guerre. Il ne commence vraiment à prendre une personnalité que lorsque l'armée se heurte aux Bédouins. Nulle sympathie pour cette race, lâche et versatile : «Il courent tousjours sus aus plus feble.» (§ 248). Pourtant Joinville s'intéresse assez à eux pour s'enquérir de leurs convictions religieuses. Il note que ce sont des Musulmans hétérodoxes, fidèles d'Ali et non de Mahomet; il rappelle leur croyance en la métempsychose. Suivent des notations plus pittoresques sur leur nomadisme, leurs grandes pelisses, leurs tentes, leurs mœurs. Cet intérêt annonce de très loin les minutieuses observations des grands voyageurs contemporains, de Plan Carpin à Rubruck ${ }^{15}$. Entortillés en leur touaille crasseuse, noirs de peau, de poils et de cheveux, les Bédouins sont, en bref, "gent hydeuse»; pire que tout, leur conviction profondément fataliste que «nus ne puet mourir que a son jour» fait des adeptes jusqu'en Occident, pour la plus grande indignation de Joinville. La curiosité tourne court devant la condamnation : on ne verra ni le roi ni ses croisés tenter de mieux comprendre ces nomades par trop éloignés de la vie des Francs. Par contraste, les Sarrasins en deviennent plus proches.

C'est lorsque le roi et Joinville tombent prisonniers entre les mains des Sarrasins que s'amorce l'ébauche d'une connaissance. La première impression est naturellement toute négative. Epée brandie, tuant tous les prisonniers pauvres dont les deux prêtres de Joinville, achevant les blessés et les malades, exigeant dans les menaces un prompt reniement, tels ils sont tout d'abord dépeints, affreux à souhait.Ils sont les traîtres assassins de leur propre soudan, ils se divisent et se déchirent entre eux, ils vont jusqu'à torturer le vieux patriarche sous les yeux de saint Louis pour contraindre le roi à un serment blasphématoire. Mais le pire est leur perfidie absolue, leur incapacité à respecter un serment, comme ils le montrent lors de l'occupation de Damiette, où ils massacrent tous les blessés. Néanmoins, si pris collectivement, les Sarrasins restent bien les «ennemis Dieu» avec lesquels un croisé ne peut discuter qu'à coups d'épée, de leur foule se détachent quelques individualités, plus complexes, presque attachantes.

Joinville n'est pas un ingrat, il rend hommage à ce sarrasin qui venait de la cour de l'empereur Fréderic et qui, on se demande bien pourquoi, décide de sauver la vie du sénéchal. Le prenant dans ses bras, car Joinville était très malade, il le porte dans un bateau où d'autres ennemis compatissants couvrent le fièvreux pour le réchauffer; et mêmè l'un d'eux va jusqu'à lui offrir un remède dont il sait l'efficacité et le sauver ainsi de l'apostume, que les croisés savaient fatal.

Après cet homme providentiel, qui, de plus, parlait franc, c'est auprès du "grand amiral des galies» qu'on mène notre sénéchal. Sur sa demande il avoue qu'il n'est point le cousin de saint Louis mais, à son visible orgueil, il peut revendiquer sa

15 Cf. A. T'Serstevens, Les Précurseurs de Marco Polo, textes intégraux, établis, traduits et commentés, Paris, Arthaud, 1959. 
parenté avec l'empereur Frédéric, décidément plus apprécié des «Ennemis Dieu» que du pape. Dès lors voici Joinville sous la protection spécial de ce notable - et là encore c'est le Sarrasin qui parle franc.Joinville et l'amiral échangeront ainsi de pieux aphorismes sur la Loi des Musulmans tandis que devant eux on tue à tour de bras les croisés malades.Est-ce ce que préconisait Saladin? s'enquiert Joinville:

Je lour fis dire, a mon Sarrazin, que il me semblait que ce n'estoit pas bien fait, car c'estoit contre les enseignemens Salehadin qui dit que l'on ne devoit nul home occire puis que on li avoit donnei a mangier de son pain et de son sel. (§ 330).

Mais le Sarrasin répond que leur maladie les a déjà condamnés.Comme les mariniers se renient à qui mieux mieux pour avoir la vie sauve, Joinville exprime une seconde fois son mécontentement et «son» Sarrasin en tombe d'accord:

Et li amiraus me fist réponse tel que il s'acordoit a moy. Que Salehadin disoit que on ne vit onques de mauvais crestiens bon sarrazin ne de mauvais sarrazin bon crestien. (§331).

Avant de quitter à jamais son protégé, l'amiral lui confie un enfant franc qui était son captif.

Mais la Providence continue de veiller sur Joinville qui en a pleine conscience. Attendant la mort en une tente au milieu de ses amis, le sénéchal voit arriver un vieillard cassé par l'âge qui s'enquiert de leurs croyances; et au rappel de la Passion du Christ, il s'écrie :

que nous ne deviens pas desconforter se nous aviens soufertes ces persecucions pour li car encore, dist il, n'estes-vous pas mort pour li ainsi comme il fu mors pour vous. Et se il est pooir de li resusciter, soiés certein que il vous deliverra quant li plaira. (§ 337).

La rencontre avec ce vieil homme à la parole prophétique fut l'un des grands moments de la croisade de Joinville. L'on sait que dans son Credo, il a pris soin de faire représenter la scène, on y voit le petit vieillard courbé, les croisés autour de lui, dont Joinville, reconnaissable à son chaperon ${ }^{16}$. S'il est peu probable qu'en ces paroles, le sénéchal ait compris que les croyants du Livre avaient avec les chrétiens quelques points d'accord, du moins la haine et l'hostilité s'étaient-elles un instant effacées entre les interlocuteurs. Plus tard, Joinville et le roi devaient discuter longuement de ces moments remarquables; et peut-être est-ce à leur lumière que saint Louis fit cette intéressante distinction entre preuz home et preudome, les premiers se trouvant aussi bien chez les meilleurs Sarrasins que chez les Chrétiens ( $\$ 560$ ).

Ennemis, certes, mais pas toujours ignobles, les Sarrasins s'émerveillent de leur côté de la largesse du roi : «par ma foi, large est li Frans quant il n'a pas barguigné.» ( $\$ 343$ ), comme de sa constance : «Il disoient que li roys estoit li plus fermes crestiens que on peust trouver.» ( $\$ 367)$; et même ils s'inclinent devant la foi de cet étranger que l'épreuve n'a fait que fortifier: «et disoient que se Mahommez lour out

16 Credo de Jean de Joinville, éd de Natalis de Wailly,Paris 1867 et L. J. Friedman, Text and Iconography for Joinville's credo, Cambridge, 1958. 
tant de meschief soufert a faire, il ne le creussent jamais.» ( $\$ 367$ ). Pendant un bref laps de temps, la compréhension aurait-elle été réciproque dans l'élan de sympathie, de pitié et d'admiration des geôliers? Ce sont ces mêmes Sarrasins enfin qui, ayant reçu l'ordre de relâcher Joinville et ses compagnons mettent leur point d'honneur à ne pas les renvoyer à jeun; et de leur servir des beignets de fromage et des oeufs durs, bariolés comme oeufs de Pâques «pour honnour de nous» (§376)...

Tout aussi imprévisible apparaît le Vieux de la Montagne qui envoie à saint Louis ce qu'on pourrait appeler une "ambassade insolente», lourde de menaces et réclamant soumission. La réponse fière et irritée du roi, entouré des grands maîtres des ordres militaires, métamorphose l'ennemi d'hier en ami, d'où assaut de politesses et de cadeaux précieux. Joinville en profite pour revenir sur les divergences religieuses des Musulmans et développe ce qu'il sait sur Ali «oncle» du Prophète. Il n'omet pas de signaler que le roi envoya auprès du Vieux de la Montagne frère Yves le Breton dans l'espoir de le convertir en prenant pour base de discussion des croyances communes, inscrites dans la Bible. Quand bien même ce fut un échec, il était nécessaire de relever ici cet essai pacifique et missionnaire en plein milieu de la croisade ( $\$ 456)$.

Le même frère Yves, dominicain «qui savoit le sarrazinois», raconta à Joinville l'histoire de la vieille femme qui marchait dans les rues avec une poèle de braises pour brûler le Paradis et une carafe d'eau pour éteindre l'Enfer ${ }^{17}$. Que Joinville ait mal compris en croyant l'anecdote contemporaine ou que frère Yves soit à l'origine de l'erreur importe peu car il y avait plusieurs siècles que «Abi'a al Adawiyya avait ainsi prêché le pur amour dans les rues de Basra mais il faut remercier (Joinville) d'avoir fait connaître cette perle à l'Occident» ${ }^{18}$;

Ces pas hésitants qui rapprochent l'ennemi de naguère se redoublent en la fin du récit par l'esquisse d'un identique mouvement chez les Sarrasins. C'est encore une fois par la bouche d'un vieillard prophétique que seront énoncées des vérités qui pourraient faire rêver de tolérance.Jean li Ermins, artillier du roi, se vit un jour apostropher par un Sarrasin qui lui demanda s'il était chrétien. Sur sa réponse positive, le vieil homme le traita de pécheur:

Lors li dist Jehans li Ermins que il se devoit bien taire des pechiez aus crestiens, pour les pechiez que li Sarrazin fesoient qui mout sont plus grant. (\$447).

S'ensuit un petit cours de théologie où le vieillard, très courtoisement, explique au chrétien que leurs péchés sont infiniment plus graves que ceux des Musulmans, puisqu'ils les commettent en pleine conscience :

\footnotetext{
17 Cf. sur ce sujet J. Monfrin, «Joinville et l'Orient», L'écrit dans la société médiévale, Paris, CNRS, 1991, pp. 259-267.

18 M. Th. d'Alverny, La connaissance de l'Islam dans l'Occident médiéval (recueil d'articles), Variorum, Aldershot, 1994, la citation se trouve dans «La connaissance de l'Islam au temps de saint Louis», p. 238. Cf. aussi l'article précédent «La connaissance de l'Islam en Occident du $\mathrm{IX}^{\mathrm{e}}$ au milieu du XII ${ }^{\mathrm{e}} \mathrm{s}$.
} 
Vous congnoissiés quant vous faites le bien et quant vous feites le mal. Dont Diex vous sait pejor grei d'un petit pechié quant vous le faites que il ne fait a nous d'un grant qui n'en cognoissons point. (\$ 448).

Et il conclut en rappelant que, pour les fidèles de Mahomet, les ablutions à l'eau peu avant la mort suffisent à purifier. Même si ces affirmations peuvent paraître simplistes et caricaturales, il faut savoir gré à Joinville et à son roi d'avoir tenté cet essai de dialogue.

A travers le portrait de Musulmans divers et pas toujours condamnés, La Vie de saint Louis nous permet de deviner que la cohabitation en Terre sainte conduisit sans doute saint Louis à s'informer sur ceux qu'il était venu combattre.L'esprit de croisade, si l'on entend par là "guerre sainte», "guerre à outrance» pouvait bien mouvoir les foules, déjà naguère et plus encore en ce milieu du XIII ${ }^{\mathrm{e}}$ siècle, c'était l'opinion des Poulains qui prévalait quand venait le temps des trêves et de l'installation.

III - Un saint roi croisé et/ou martyr.

Peu après la mort de Louis IX, ses successeurs et ses amis, tant ecclésiastiques que laïcs, entreprirent de faire officiellement canoniser ce roi qui avait su pour eux incarner les plus purs idéaux de leur foi et de leur pays ${ }^{19}$.

Si Joinville ne fut sans doute pas de ceux qui «poussèrent» à la canonisation, il est bien évident que son témoignage, qui dura deux jours pleins, fut capital pour le procès. On peut cependant se demander quels traits de la sainteté du roi emportèrent la conviction des contemporains, comme on a hésité pour le titre du livre de Joinville, La Vie saint Louis de N.Corbett ayant délibérément choisi le registre hagiographique, alors que l'Histoire de saint Louis, retenu jadis par Natalis de Wailly, ressortissait davantage de la biographie et de l'Histoire. Joinville scinde son livre en deux parties dont l'une, la croisade, retrace plus particulièrement les hauts-faits du saint Roi-Chevalier, tandis que l'autre se consacre à ses enseignements. quelque peu cléricaux.

Parmi les hommes d'Eglise, il y avait déjà longtemps que l'idée de croisade était battue en brèche. Si les critiques d'Adam de Perseigne sont peut-être celles d'un cistercien aigri par les événement de Zara, d'autres religieux avaient simplement une plus haute idée de la conversion. Dès le XII ${ }^{\mathrm{e}}$ siècle, Pierre le Vénérable, en faisant traduire le Coran, avait montré sa conscience «de l'ignorance profonde des Occidentaux vis à vis de l'Islam; et, soucieux du salut de l'âme des sectateurs de Mahomet, réalisait qu'il pouvait exister des méthodes d'apologétique plus souhaitables que les coups d'estoc des croisés... Pour arriver à les convaincre, il fallait d'abord essayer de les comprendre» 20 .

Plus près de saint Louis, François d'Assise n'était-il pas parti en Syrie en 1221, armé de sa seule ferveur pour rencontrer les Musulmans, qui avaient d'ailleurs respecté sa douce et folle entreprise mais lui avaient refusé un martyre dont il rêvait

19 K. Uitti, art. cit. , p. 380.

20 M. Th. d'Alverny, op. cit. "Deux traductions latines du Coran au Moyen Age», p. 70. 
peut-être en secret.Or le franciscain Hugues de Digne que saint Louis rencontra à Hyères et bien d'autres frères prêcheurs faisaient l'habituelle compagnie du roi qui ne pouvait ignorer la pacifique croisade du Poverello.

"On peut se demander, remarque B.Guillemain ${ }^{21}$, si comme son contemporain, Frédéric II, et dans un environnement tout différent, saint Louis n'a pas pensé entre ses deux croisades que sa mission était de redresser, de purifier, de préparer son peuple à une imminente fin des temps, comme s'il était un roi des derniers jours». Et ce n'est pas le milieu franciscain, tout pénétré de millénarisme, qui aurait contredit le roi en ces pensées.

Un vent d'eschatologie souffle parfois sur La vie de saint Louis. Cela est tout particulièrement sensible lorsque Joinville narre la double ambassade des Tartares. La première se déroule à Chypre :

Li grans roys des Tartarins...entre les autres li manda que il estoit prez de li aidier a conquerre la Terre Sainte et a delivrer Jherusalem de la main aus Sarrazins. ( $§$ 133).

Mais ces belles prémices se trouvent vite confrontées à la réalité des faits et, tout d'abord, pour les messagers partis dans l'allégresse, c'est la découverte de «plusors citez que il avoient destruites et grans monciaus d'os de gens mors» ( $\$$ 472-473); puis ce sont les nouvelles que ce peuple, naguère sujet de l'empereur de Perse et du Prêtre Jean, se sont rebellés et ont écrasé leur maître, objet du long espoir des chrétiens. Les Tartarins, nourris de viande crue, atendrie sous la selle et consommée à sa putréfaction, se revèlent un peuple insolent et dangereux, prompt à demander au roi tribut et soumission. Mais pouvait-il en être autrement lorsqu'on se souvient de leur origine?

Il estoient venu et concreei d'une grant berrie de sablon la ou il ne croissait nul bien. Cette berrie commençoit a uns tres grans roches merveillouses qui sont en la fin dou monde devers Orient, lesqueix roches nulz hons ne passa onques... et disoient que leans estoit enclos li peuples Got et Margoth qui doivent venir en la fin dou monde quant Antecriz venra pour tout destruire. (\$ 473).

Ayant compris que cette race sanguinaire et suffisante est tout simplement celle des fourriers de l'Antéchrist, saint Louis «se repenti mout quant il y envoia» ( 492).

Dans cette atmosphère de fin des temps, on comprend mieux le sens profond de l'exemplum prêté à Guillaume d'Auvergne sur ce prêtre ravagé par son scepticisme touchant la Présence Réelle: le monde est une citadelle assiégée où rôde le Démon (§ 46). Et Joinville en avait été averti dès l'enfance par sa mère qui lui avait recommandé de toujours signer ses lèvres avant de parler, pour se mettre sous la protection de l'Esprit (§ 435).

Joinville, nous le savons, est providentialiste, il croit à une présence de Dieu continuellement révélée par des signes. Il lui faut pourtant expliquer l'échec de la croisade tout comme il lui faut justifier son refus de repartir.Pour l'échec, la réponse

21 B. Guillemain, «Le sens de l'histoire au XIII ${ }^{ }$, 1274, année charnière,mutations et continuités, édition CNRS, Paris, 1974, pp. 881-886, (citation p. 886). 
est celle dont les hommes du Moyen Age usent depuis la seconde croisade ce fut la faute des péchés de l'armée :

Autant puet dire Nostre Siress de nous comme il dist des fiz Israel la où il dist Et pro nichilo habuerunt terram desiderabilem. Et que dist il après ? Il dist qu'il oublierent Dieu qui sauvez les avoit. (§ 166).

Ces péchés sont détaillés : oubli par le roi lui-mème d'une «bonne coutume» ancienne à Damiette avec pour conséquence un enchérissement terrible des denrées, l'avarice et la mollesse des seigneurs, le relâchement des mœurs que souligne la présence des «folles femmes», habituelles compagnes et coupables toutes désignées des troupes militaires. Le résultat est que le nombre des hommes devient insuffisant alors que l'Histoire a appris aux croisés que la foi remplace avantageusement les foules, comme le rappelle le vieux musulman à Jehan li Ermins :

Mout vous devez haïr entre vous crestiens, que j'ai veu tel fois que li roys Baudouins de Jerusalem qui fu mesiaus desconfist Salehadin; et n'avoit que trois cens homes a armes et Salehadins trois milliers ; or estes tellement menei par vos pechiés que nous vous prenons aval les chans comme bestes. (\$ 466).

Argument irréfutable : le péché animalise et rend vulnérable; mais l'absence de péché suffit-elle à la sainteté ?

La sainteté du roi, Joinville ne la met pas en doute, même si, du vivant du roi, il voyait une bonne plaisanterie dans la demande des Arméniens qui voulaient contempler et adorer de vivo le «saint roi»; et saint Louis d'en rire à gorge déployée avec son sénéchal. Pourtant, à la lumière des lignes finales où Joinville réclame précisément les os de son roi pour reliques en sa chapelle, cette anecdote acquiert une certaine densité douloureuse. Mais l'idée de la sainteté reste distincte du profond amour du sénéchal pour Louis IX. Que le roi soit saint, c'est Dieu même qui l'a signifié.

Il l'a manifesté toutes les fois où saint Louis prend des décisions inconsidérées et aventureuses qui «mettent son cors en aventure de mort», décisions dont Joinville spécifie bien qu'elles se réalisèrent à chaque fois contre l'avis du conseil royal, et toutes décisions advenues au temps de la croisade. Ainsi de celle de Damiette où il courut sur la plage contre les Sarrasins et où ses compagnons n'eurent que le temps de l'arrêter; à la Mansourah où, épuisé par la dysenterie, il refusa de s'enfuir par le Nil, ce qui lui valut d'être fait prisonnier: «Il ne vout onques nullui croire ; ançois dist que son peuple ne lairoit il ja mais feroit il tel fin comme il feroient.» (\$ 10). Ainsi encore à Acre; appuyé par Joinville, il décide de demeurer durant quatre ans outremer, alors que ses troupes étaient des plus réduites; mais par une grâce toute spéciale, ils ne se virent pas attaquer par des Turcs, infiniment supérieurs en nombre ; enfin, la dernière fois, quand il prend la décision de rester sur un bateau à demi éventré parce que des marchands âpres au gain lui avaient confié qu'en pareille circonstance ils n'auraient jamais abandonné leur cargaison, dussent-ils en mourir. Certes cela évite un long séjour sur des îles d'où les pauvres croisés avaient un mal fou à s'en aller par défaut d'argent; mais c'était se confier à la mer aux vents imprévisibles - ou à Dieu, en une sorte d'ordalie, que l'événement, depuis longtemps 
terminé heureusement, vient justifier a posteriori pour Joinville. C'est donc l'heureuse issue de toutes ces folies qui montre de façon éclatante la sainteté de l'Elu.

Joinville, qui n'hésite pas à saluer en chevalier la belle prouesse de son roi, ne condamne pas l'entreprise armée que fut la croisade; mais il a des mots très durs contre le second départ qui devait être fatal à Louis : le roi était trop affaibli par la maladie dès avant ce voyage sans lequel il eût pu survivre longtemps, pour le plus grand profit de son peuple; ses compagnons acquiescèrent du bout des lèvres ; enfin ce fut une «croisade de petit esploit», signe que Dieu ne la demandait point. Quant aux raisons alléguées par le sénéchal à son roi et à son suzerain de Champagne pour justifier sa demurée, elles sentent fâcheusement le prétexte. Joinville ne voulait pas repartir; on peut se demander si, en 1267 , la croisade encore proche ne brillait pas de moins belles couleurs dans son souvenir, que lors de son extrême vieillesse, une fois son roi dûment sanctifié.

Saint Louis vécut les yeux constamment fixés sur le modèle christique. Et sa mort même, bras en croix sur un lit de cendre, râlant dans son agonie le nom trop aimé de Jérusalem, est pour Joinville une mort de martyr:

Et de ce me semble il que on ne li fist mie assez, quant on ne le mist ou nombre des martirs pour les grans peinnes qu'il souffri ou pelerinaige de la croiz par l'espace de siz ans que je fu en sa compaignie, et pour ce meismement qu'il ensui Nostre Signours ou fait de la croiz. Car se Diex morut en la croiz aussi fist il car croisiez estoit il quant il mourut a Thunes. (\$5).

Martyr donc, il a mérité la sainteté par cela même que toutes les ombres qui entourent les croisades, et dont Joinville fut assez conscient pour ne pas repartir, ont été effacées par le sacrifice expiatoire, point d'orgue posé par Dieu à une existence à lui consacrée mais aussi placée tout soudain hors de l'humanité ordinaire et peutêtre même de l'imitation - ce fut la dernière croisade... Comme Jacques de Voragine, comme ses contemporains, et le roi sans doute le tout premier, Joinville imagine d'abord la sainteté sous les traits des saints des premiers temps; mais comme Voragine, le sénéchal les admire sans penser pour autant qu'on puisse les imiter ${ }^{22}$, d'ailleurs ils font partie du mythique passé, meilleur mais achevé. Ainsi s'expliqueraient les réticences, les silences, voire les contradictions qu'on trouve ici et là dans la Vie de saint Louis touchant l'avis profond de Joinville sur la croisade, avis qu'il se refusait sans doute à se formuler clairement.Il aurait suivi jusqu'au bout de l'orient celui qu'il aimait mais à «sa mort» néanmoins, il «n'estoit mie»... Mort inexplicable, mystérieuse, admirable du martyre accepté.

A choisir la voie d'Egypte, plus que par l'avis de son frère préféré, saint Louis se sentit-il poussé par tous les souvenirs que l'Evangile attache à cette terre, par la présence du Chrême et du Baume qui feraient de lui, à en conquérir le réceptacle, un autre David ${ }^{23}$, doublement consacré ? Pour le chevalier de Champagne que fut notre sénéchal, la croisade avec ses beaux faits d'arme a quelque chose de l'épopée ; mais

22 Cf. J. Boureau, Le système narratif de Jacques de Voragine, Paris, 1984.

23 Cf. J. P. Albert, Odeurs de sainteté, la mythologie chrétienne des aromates, Paris, 1990. 
les hommes s'avèrent, sauf dans leurs rêves, bien trop pécheurs pour être dignes de libérer la Sainte Terre. De la grande espérance un peu folle qui emporta son ami, Joinville ne devait conserver que l'amère et merveilleuse consolation d'avoir aimé un saint, victime que Dieu lui-même avait élevée aux yeux de ses contemporains en la broyant dans ses meules.

Marie-Geneviève Grossel 